\title{
Que sait-on des jeux de société « modernes »?
}

What do we know about "modern" board games?

\section{Vincent Berry}

\section{(2) OpenEdition}

Journals

Édition électronique

URL : http://journals.openedition.org/sdj/2796

DOI : $10.4000 /$ sdj.2796

ISSN : 2269-2657

\section{Éditeur}

Laboratoire EXPERICE - Centre de Recherche Interuniversitaire Expérience Ressources Culturelles Education

\section{Référence électronique}

Vincent Berry, "Que sait-on des jeux de société « modernes »? », Sciences du jeu [En ligne], 14 | 2021, mis en ligne le 06 mars 2021, consulté le 06 avril 2021. URL : http://journals.openedition.org/sdj/2796 ; DOI : https://doi.org/10.4000/sdj.2796

Ce document a été généré automatiquement le 6 avril 2021.

\section{c) (i) $९$}

La revue Sciences du jeu est mise à disposition selon les termes de la Licence Creative Commons Attribution - Pas d'Utilisation Commerciale - Pas de Modification 4.0 International. 


\title{
Que sait-on des jeux de société «modernes »?
}

\author{
What do we know about "modern" board games?
}

\section{Vincent Berry}

1 Si l'on accepte une définition large - et donc discutable - du jeu de société, entendu comme un ensemble de pratiques ludiques se déroulant le plus souvent autour d'une table et qui supposent l'usage de règles et d'un matériel dédié ou adapté, ce domaine connait depuis plusieurs décennies un développement important. ${ }^{1}$ Aux jeux «traditionnels » anciens (Dames, Échecs ou Petits-Chevaux) et aux franchises industrielles établies telles que le Monopoly (Hasbro, 1935) ou le Cluedo (Hasbro, 1949) s'ajoute en effet une galaxie en expansion de jeux en moyenne moins diffusés, mais dont certains réalisent parfois une percée auprès du grand public. On peut penser à titre d'exemple aux Loups Garous de Thiercellieux (Lui-même, 2001) ou à Jungle Speed (Asmodée, 1996), titres initialement auto-édités et vendus à plusieurs millions d'exemplaires dans le monde. Sur les vingt dernières années, l'offre ne cesse de croître : en 1996 on comptait près de 200 nouveaux titres édités sur le marché français - hors extensions de jeux existants et rééditions - ; en 2017, plus de 600 (Berry, 2017). Conjointement, le nombre de nouveaux éditeurs augmente. On en recense près d'une centaine, parmi lesquels de nombreux créateurs indépendants aux franges d'un marché dominé par des entreprises multinationales (Berry \& Roucous, 2020).

\section{Le développement du jeu de société}

Du côté des pratiques, les évolutions sont plus difficiles à cerner car les données sont rares. On peut toutefois observer quelques transformations notables en France à travers la lecture de certaines statistiques publiques qui, malgré le caractère large et parfois flou des catégories sur le jeu qui y sont mobilisées, convergent toutes ou presque vers une augmentation des activités ludiques dans les dernières décennies. L'analyse des différentes vagues de l'enquête "Emploi du temps" (EDT) de l'INSEE révèle ainsi qu'en l'espace de 40 ans les «activités récréatives », qui incluent le jeu de 
société, mais pas seulement ${ }^{2}$, ont continuellement augmenté. Le temps qui leur est alloué s'est vu multiplié par $4:$ « de 1 heure par semaine en 1974, à près de 4 heures en $2010 »$ (Brousse, 2015, p. 105).

Dans un contexte d'augmentation générale du temps libre à partir des années 1980 et jusque dans les années 2000 (Chenu \& Herpin, 2002), cette progression tient principalement à l'essor de l'informatique et aux jeux vidéo dont l'usage passe de quelques minutes par semaine en 1986 à 35 minutes en 1998 pour atteindre 2,6 heures en moyenne en 2010 (Brousse, 2015). Cependant, loin de s'effacer devant leur cousin numérique, les pratiques de jeux de société, qu'il s'agisse de cartes, de dés, de pions, de figurines, d'argent ou de plateaux, se sont non seulement maintenues et ont, pour certaines, augmenté. Entre 1960 et 1992, la part des budgets des ménages consacrée aux jeux de société, aux jouets et aux jeux de hasard a continuellement progressé : ils représentaient près de $5 \%$ des dépenses de «loisirs » en 1960 pour près du double en 1992 (Léger, 1994). L'analyse comparée des données des enquêtes « loisirs » de l'INSEE de 1967 et 1987 confirme cette augmentation aussi bien en termes d'usages effectifs que de fréquences (Choquet \& Valdelièvre, 1988). Estimée à $13 \%$ en 1967, la pratique hebdomadaire de jeux de société ${ }^{3}$ concerne plus de $18 \%$ des enquêtés vingt ans plus tard (Dumontier \& Valdelièvre, 1989).

Prenant le relai des enquêtes « loisirs » de l'INSEE à partir des années 1970, les données recueillies à intervalle régulier par le Ministère de la culture sur les " pratiques culturelles » observent cette progression, mais de façon plus fragmentée et parcellaire dans la mesure où, selon les éditions, le questionnaire intègre des variables différentes sur le jeu, rendant ainsi la comparaison difficile ${ }^{4}$. En 1974, $74 \%$ des enquêtés déclaraient posséder à domicile des « jeux de société » ${ }^{5}$ et $24 \%$ d'entre eux indiquaient en avoir un usage fréquent ${ }^{6}$. Sept ans plus tard, le taux d'équipement dans les foyers se situe autour de $78 \%$ pour près de $27 \%$ de pratiques fréquentes déclarées ${ }^{7}$. Les enquêtes suivantes n'interrogent plus la possession de jeux de société à domicile, mais quelques activités ludiques seulement parmi lesquelles les cartes auxquelles $43 \%$ des enquêtés déclaraient en $1989^{8}$ avoir joué dans l'année au moins occasionnellement ${ }^{9}$. En 1997, ces dernières sont agrégées à la catégorie "jeu de société $»^{10}$ et concernent $53 \%$ de l'échantillon : $21 \%$ des enquêtés déclarent des pratiques régulières dans l'année, $29 \%$ des pratiques occasionnelles, et $3 \%$ seulement pendant les vacances ${ }^{11}$. Dans la dernière édition de 2018, qui se révèle particulièrement riche et détaillée pour le domaine qui nous intéresse ${ }^{12}, 56 \%$ des personnes résidentes en France déclarent avoir joué au moins une fois à un jeu de société au cours des 12 derniers mois ${ }^{13}$.

Cette progression des pratiques observée au tournant des années 1980 par différentes enquêtes s'accompagne d'un mouvement continu de féminisation. Qu'il s'agisse de l'enquête "Emploi du temps ", de l'enquête "Pratiques culturelles» ou des vagues «loisirs » INSEE, différents domaines de la culture ludique sont concernés : les jeux de cartes, les jeux de plateaux (dames, échecs), les jeux de chiffres et de lettres (les mots croisés), mais aussi, dans une moindre mesure, quelques activités ludiques traditionnellement masculines telles que les jeux d'argent (poker, casino, loterie) et les paris hippiques et sportifs (Yonnet, 1999). Ces évolutions concernent le domaine du jeu, mais pas seulement. Elles s'inscrivent dans un processus plus large de "féminisation des loisirs » qui tient à la fois à l'augmentation du temps libre des femmes et à un début de convergence entre les emplois du temps féminins et masculins ${ }^{14}$ (Brousse, 2015). 
6 À ce mouvement d'augmentation et de féminisation des pratiques ludiques qu'observent les enquêtes publiques au tournant des années 1980 correspond le développement d'une nouvelle offre de jeux de société qu'il s'agisse des premiers « jeux électroniques $»^{15}$, des jeux de rôles (Peterson, 2012) ou encore du renouveau des Wargames (jeux de guerre) commerciaux (Bourguilleau, 2020) et des jeux de figurines à l'image du développement de la gamme Warhammer par la société Games Workshop. Durant cette période, les modes de diffusion, de production et de commercialisation, mais aussi les contenus se transforment et marquent une rupture avec leurs formes habituelles et traditionnelles (Brougère, ce numéro [1979]). Caractérisé par une fictionnalisation accrue de ses thèmes et par des emprunts de plus en plus fréquents à la science-fiction et au merveilleux (Dauphragne 2007), un nouveau régime du jeu de société s'impose. Il devient l'objet d'une presse spécialisée et de clubs (Berry, 2021) à mesure que se constitue un public de jeunes adultes passionnés et de collectionneurs « sérieusement investis » dans ce hobby (Rogerson \& Gibbs, 2018). Dans sa nouvelle forme marchande et éditoriale, le jeu de société ne renvoie plus seulement à un divertissement enfantin et familial (Whitehill, ce numéro), mais à un loisir dont une frange d'amateurs travaille à souligner sa valeur culturelle (Woods, 2009). Ces mêmes années 1980 observent dans le champ de l'éducation une accélération du développement des ludothèques en France (Legendre, 2015) qui font du jeu de société, mais aussi du jouet, le cœur de leur action sociale et de leur projet éducatif (Brougère et Roucous, 1998).

7 Si les données statistiques dont nous disposons montrent ainsi à grands traits une progression des pratiques de jeu de société, une augmentation de l'offre ainsi qu'une évolution de son statut culturel sur les cinquante dernières années, les catégories mobilisées dans les enquêtes sont souvent larges et imprécises. En conséquence elles masquent la complexité de ces transformations ainsi que la diversité des publics et l'hétérogénéité des usages que recouvre le terme. De la partie de Belote entre amis au bistrot ou à l'usine, au Monopoly familial à la maison ou en ludothèque, en passant par les « soirées jeu » entre adultes dans des clubs ou dans des cafés-jeux, le monde du jeu de société est divers et changeant. De nouvelles pratiques et formes sociales peuvent apparaitre à mesure que d'autres s'effacent. De la même façon, le mot « jeu de société » et ses usages demeurent toujours ambigus. Les représentations qui lui sont attachées peuvent évoluer et différer selon les périodes et les enquêtes de sorte que «dans un groupe humain donné, pris à un moment de son histoire, il y a des choses auxquelles on confère le statut de jeux (et d'autres auxquelles on le refuse) »(Henriot, 1978, p. 17).

8 Quelles que soient les limites propres à chacune d'entre elles, les grandes enquêtes publiques convergent cependant sur une augmentation de certaines pratiques et soulignent une implantation croissante et durable de quelques-unes d'entre elles dans l'espace culturel des Français. À la question maintes fois posée par les médias à l'occasion de Noël de savoir si «les gens jouent plus qu'autrefois?", la recherche ne peut y répondre de façon générale et tranchée. Loin des thèmes de l'« invasion du jeu » et de l'« emprise du ludique " sur le monde social régulièrement prophétisés par des essayistes, l'étude empirique des usages révèle que quelques formes sociales et certains genres de jeux se sont progressivement imposés et font aujourd'hui partie de l'horizon des loisirs ordinaires. Comme s'y emploie le premier article de ce numéro (Berry \& Coavoux), une sociologie minutieuse et détaillée des pratiques, des publics, des lieux et des types de jeux reste à faire. 


\section{Le jeu de société dans les sciences humaines et sociales}

9 En dépit de sa progression en termes d'offres et de pratiques sur les dernières décennies, le jeu de société demeure un domaine culturel relativement peu abordé par les sciences sociales. Alors qu'un champ international de recherches historiques - les Board Games Studies ${ }^{16}$ - s'est constitué depuis près de 30 ans autour de l'étude des jeux traditionnels, l'analyse de ses formes contemporaines se révèle comparativement moins importante. Elle se concentre le plus souvent sur des secteurs particuliers comme le jeu d'échecs (Wendling 2002, Borzakian 2007, Fine 2015), le jeu de rôles (Trémel, 2001; Fine, 2002 ; Lizé, 2004 ; Caïra, 2007 ; Dauphragne 2008; David, 2016 ; Perier, 2017) ou encore sur le domaine des jeux d'argent et de hasard (Martignoni 2011, Amadieu, 2013, Redon \& Lebeau, 2020) tels que le Poker « en dur » ou en ligne (Brody, 2011 ; Pastinelli, 2011) et les tripots clandestins (Feyrs, 2002 ; Peidallu, 2012). Notons également que, contrairement aux jeux d'argent (Trespeuch, 2013) et aux jeux vidéo (Benghozi \& Chantepie, 2017; Zabban, 2020), l'industrie est peu étudiée dans la littérature scientifique, à l'exception de travaux sur le secteur de masse aux États-Unis (Whitehill, 1992; Peterson, 2012) et de quelques enquêtes sur les trajectoires des auteurs et des éditeurs en France (Berry \& Roucous, 2020) et sur le secteur des « indépendants » (Tyni, 2020).

Qu'il s'agisse d'une pratique confidentielle comme le jeu de rôle ou d'un domaine socialement visible comme les jeux d'argent, ces enquêtes s'attachent le plus souvent à analyser les modes d'organisation de ces mondes sociaux, mais aussi les enjeux politiques, sanitaires voire éducatifs. Fréquemment, les pratiques de jeux de société y sont abordées comme des indicateurs de transformations sociales plus larges dont ils sont l'expression. On peut ainsi penser au travail de Paul Yonnet qui observe au tournant des années 1980 une diminution des pratiques ludiques populaires et particulièrement des paris hippiques et sportifs. Le déclin de cette «France des tiercéiste " est à mettre en relation avec un double mouvement: d'un côté une "reconsidération des rôles sexuels ", liée aux mouvements féministes et au progrès sociaux acquis durant la décennie précédente dans le domaine de l'égalité des droits et, de l'autre, un processus de désindustrialisation qui voit la fermeture progressive des usines. Cette «crise généralisée » de la classe ouvrière et des formes traditionnelles de la masculinité se traduit selon le sociologue par le déclin des jeux de paris sportifs, mais aussi par la baisse de la fréquentation des cafés, haut lieu des pratiques ludiques populaires et masculines, au profit du développement des loisirs d'intérieurs, au premier rang desquels la télévision s'impose rapidement comme une "féroce consommatrice de temps non travaillé » (Yonnet, 1999, p. 259).

11 Aux analyses de Yonnet on peut rapprocher les travaux de Florence Weber sur la culture ouvrière dans lesquels elle observe, au milieu des années 1980, une appétence croissante pour les jeux d'argent, les loteries et les jeux de tirage et de grattage. Elle met en parallèle l'essor de ces pratiques ludiques avec la précarisation grandissante des classes populaires au sortir des Trente Glorieuses. Le succès de ces jeux lui apparait comme une « ultime tentative pour restaurer, par le recours au hasard, l'égalité devant le sort, après la chute de la méritocratie scolaire républicaine » (2008, p. 208). Dans une perspective proche, mais dans un tout autre domaine, celui du jeu de rôle, Laurent 
Trémel (2001) met en relation l'avènement de ce nouveau jeu de société avec les évolutions sociales et économiques des années 1980 et 1990 marquées par le (sentiment de) déclassement d'une jeunesse aux prises avec la précarité et le chômage de masse. Le chercheur perçoit dans le succès de ces nouveaux dispositifs ludiques une forme de reclassement symbolique qui, en offrant des "carrières ludiques", permet de compenser dans la fiction ce que le monde social ne permet pas, ou plus, d'offrir en termes d'intégration.

Dans les rares enquêtes qui en font ainsi l'étude, le jeu de société est souvent mobilisé sous l'angle d'un indicateur de transformations sociales, culturelles et démographiques, mais aussi au prisme des sociabilités qui donnent corps à sa pratique. Analysant l'évolution statistique des pratiques culturelles des seniors au lendemain de la Seconde Guerre mondiale, Jean-François Bikel, Christian Lalive d'Épinay et Nathalie Vollenwyder remarquent la place grandissante que le jeu de société occupe progressivement au sein du temps libre, et plus particulièrement les cartes. Confirmant en partie la thèse du développement d'une « civilisation du loisir » (Dumazedier, 2018 [1962]), cette augmentation témoigne d'une amélioration objective des conditions de vie des personnes âgées, mais aussi de l'affirmation d'une nouvelle norme culturelle qui accorde la part belle "aux loisirs, à l'épanouissement personnel, à l'expressivité corporelle et à diverses formes de participation sociale »(Bikel et al., 2005, p. 156).

Dans une perspective tout aussi attentive aux évolutions des pratiques ludiques dans le temps, Samuel Coavoux et David Gerber mettent en relation le succès de certaines formes d'activités ludiques avec les sociabilités dans lesquelles elles se déploient. À partir des données sur les pratiques de jeu vidéo et de jeux de société issues de l'enquête Ludespace (Ter Minassian et al., 2021), ils observent une différenciation et une évolution des activités ludiques tout au long de vie qu'ils expliquent notamment au regard des fonctions distinctes que le jeu occupe selon les groupes sociaux où il se pratique. Les jeux vidéo, dont l'intensité et la diversité des usages se révèlent les plus élevées chez les adolescents (Octobre et al., 2010), sont un important point d'appui aux "sociabilités électives" et à l'émancipation des jeunes de la sphère domestique. À l'inverse, moins concentrée sur des intervalles d'âges précis, la pratique des jeux de société apparait comme plus durable dans le temps et servir plus spécifiquement de socle « aux sociabilités intra-familiales » (Coavoux \& Gerber, 2016).

Parallèlement à ces approches de type sociométrique, quelques travaux en géographie s'intéressent aux jeux de société en s'attachant plus particulièrement à comprendre leurs modes de diffusion sur les territoires. À partir d'une analyse croisée des données des fédérations de jeux en France (Bridge, Dames, Échecs, Go, Scrabble et Tarot), Manouk Borzakian $(2010,2015)$ observe certaines spécificités régionales et départementales, les Dames dans le nord du pays par exemple, qu'il met en relation avec les caractéristiques socio-démographiques des régions (ouvrières pour certaines), mais aussi avec des contextes politiques et des trajectoires, parfois singulières, de personnalités politiques locales à l'origine du succès d'un jeu sur un territoire. Dans une perspective proche, Marie-Françoise Fleury et Hervé Thery étudient la circulation du Monopoly dans le monde en examinant l'ensemble des éditions locales ou internationales (mais aussi les contrefaçons) et leur évolution. L'analyse des rues et de leurs adaptations selon le pays de diffusion permet d'observer la force des relations historiques entre nations et la persistance (ou non) de certains liens coloniaux. Le « succès mondial » du plus célèbre des jeux des Frères Parker se révèle selon les géographes « un excellent indicateur des 
zones d'influence des principales puissances économiques » (Fleury et Thery, 2002, p. 6). ${ }^{17}$

15 Si beaucoup de travaux voient ainsi dans le jeu de société l'expression de problématiques sociales, historiques ou territoriales, quelques recherches y circonscrivent l'essentiel de leur questionnement. De façon comparable au champ de recherche international et interdisciplinaire consacré au jeu vidéo, les «Games Studies" (Zabban, 2012), une littérature académique s'est développée depuis une dizaine années pour en faire l'objet central de l'analyse ${ }^{18}$. Il s'agit moins de considérer le jeu de société comme indicateur, mais de comprendre les logiques spécifiques au média : les formes d'expérience et d'interactions qu'il produit, la diversité des modes " d'engagement ludique » des joueurs (Caïra, 2010) ou encore les relations entre fiction et identité (Barbier, 2012). Dans le monde anglo-saxon, cette perspective "gamecentric", centrée sur le jeu, est plus marquée encore. S'inscrivant dans des perspectives appliquées de Game Design (conception de jeu), le jeu de société est étudié dans ses dimensions techniques et sa "matérialité » qui participent pleinement du plaisir et de l'engagement des joueurs (Rogerson et al., 2016). À travers des approches de type sémiologique ou narratologique, il s'agit de s'intéresser à la façon dont le jeu de société produit des expériences singulières et, par extension, d'analyser son potentiel éducatif et ses usages dans le champ de la formation (Bayeck, 2020).

\section{Présentation du numéro}

16 Au terme de cette rapide revue de la littérature, qui ne prétend pas à l'exhaustivité, il reste difficile à ce jour de répondre avec précision à des questions relativement simples, à certains égards naïves, mais au demeurant essentielles : qui joue aux jeux de société ? À quels jeux ? Avec qui ? La multiplication actuelle de l'offre correspond-elle à une diversification des usages? Comment sont-ils appropriés par leurs publics? Comment est structurée l'industrie des jeux de société ? L'objectif de ce numéro de Sciences du jeu est de proposer les premières briques empiriques d'une réflexion visant à combler ce manque et de contribuer à une connaissance nouvelle dans le champ des études sur le jeu. Sans bien sûr prétendre répondre à l'ensemble de ces questions, le présent numéro souhaite apporter quelques éléments de réponse.

17 Le premier article de Vincent Berry et Samuel Coavoux propose un cadrage sociologique des pratiques de jeu de société en France. À partir d'une enquête statistique inédite, il étudie les formes sociales du jeu de société. Des usages en famille jusqu'aux activités des passionnés en passant par les parties occasionnelles entre adultes, le spectre des pratiques est décrit au regard des propriétés sociales des joueurs. Loin d'être résiduel, le jeu de société apparait comme un loisir important et se révèle, à ce titre, inégalement distribué. Construite autour d'un « test de culture ludique » visant à mesurer à la fois l'expérience - le fait d'avoir joué au moins une fois dans sa vie - et la connaissance de l'offre, l'étude souligne l'importance des effets d'âge, de genre, de milieux sociaux, mais aussi de générations. Si un public adulte de passionnés s'est durablement constitué dans les dernières décennies et si certaines formes de jeu, notamment en famille, apparaissent plus importantes chez les classes moyennes et supérieures, le modèle de l'omnivorisme culturel structure l'ensemble des pratiques: les différences portent moins sur la fréquence et l'intensité de l'activité que sur les titres et les genres privilégiés par les usagers, entre, par exemple, les «jeux 
traditionnels » d'un côté et les «jeux modernes » de l'autre. Au sein de ces ensembles d'autres différences, entre les jeux eux-mêmes, viennent distinguer les publics.

De façon plus qualitative et située, l'article de Jean-Emmanuel Barbier s'intéresse aux usages des passionnés en examinant un moment crucial de la pratique, la transmission des règles. Sur la base d'une analyse des interactions de joueurs au sein de «soiréesjeux » et de clubs, l'auteur mène une analyse didactique du jeu de société en étudiant en détail les conditions de cette transmission. Loin de se réduire à des consignes qu'on lit dans un manuel, l'explication constitue un moment important d'une partie. Marque d'une expertise et d'une appartenance à la communauté des «ludistes", cet apprentissage apparait d'abord, pour celui qui explique, comme un difficile et exigeant " exercice ludologique ». Il s'agit de donner des instructions tout en créant un climat de bonnes réceptions qui se révèleront importantes dans l'expérience générale de la partie. Objet de conflit, de tensions et d'humour, la transmission s'appuie sur des ressources diverses : le livret de règles bien sûr, mais pas seulement. La matérialité des jeux et la manipulation des éléments de la boite sont autant de prises dont les joueurs se saisissent pour créer une situation d'apprentissage efficiente. Cette analyse des situations amène le chercheur à interroger la place croissante de tutoriels numériques, de vidéo sur Internet et de jeux numérisés sur tablettes qui permettent, comme pour le jeu vidéo, un apprentissage plus inductif. Ces dispositifs modifient selon le chercheur le mode habituel de transmission dans la communauté des passionnés et plus largement l'organisation du secteur qui voit apparaitre de nouveaux intermédiaires: des professionnels de l'explication de règles.

19 L'article de Vinciane Zabban, Nicolas Piñeros et Thimothée Roux interroge également ce que le numérique fait au jeu de société, mais du côté de la production. En s'intéressant aux plateformes de financement participatif sur Internet, les auteur.e.s étudient l'impact de ces nouvelles formes d'édition sur le monde professionnel. À partir d'une enquête monographique d'une campagne de financement et d'une série d'entretiens, l'article rend compte de l'ensemble des activités nécessaires à la production d'un jeu et des difficultés auxquelles les acteurs doivent faire face et qui supposent le plus souvent un apprentissage "sur le tas». En marge du secteur traditionnel et en quête d'un modèle économique soutenable à ses débuts, le «financement par la foule " s'est progressivement professionnalisé pour s'imposer comme une norme dans le champ des éditeurs installés. Loin de bouleverser l'ordre établi, cette modalité de production se développe principalement sur des produits de niche (jeux de rôles, jeux de figurines et "gros jeux») et crée une forme très particulière de relation marchande entre consommateur et concepteur « en permettant aux premiers de s'engager dans la construction d'un marché de qualité ». Si elles permettent de financer des projets difficilement viables sur le marché de masse, les plateformes de financement fonctionnent aussi comme des dispositifs d'enrôlement des publics dans la construction conjointe de ressources, matérielles et symboliques, nécessaires à l'existence de jeux de niches, que contribuent à faire exister les pratiques des passionnés et que vient ici renforcer le numérique.

Que l'intérêt soit porté aux éditeurs, aux publics ou aux pratiques des passionnés, les trois premiers articles du numéro mettent en évidence des transformations éditoriales, sociologiques ou économiques du secteur sur les dernières décennies. Pour éclairer ces évolutions, celles notamment qui marquent le passage des jeux traditionnels comme les Petits Chevaux aux jeux contemporains comme le Monopoly, nous avons fait le choix de 
rééditer un article de Gilles Brougère de 1979, augmenté d'une présentation originale. $\mathrm{Au}$-delà de son caractère pionnier dans la recherche francophone, ce texte nous semble particulièrement important pour tout chercheur dans le domaine. Observant un ensemble de changements à la fin des années 1970, aussi bien dans ses modes de commercialisation que dans ses contenus, l'article propose plusieurs pistes d'analyses pour comprendre les ruptures opérées par le jeu de société moderne. À l'inverse d'un jeu de cartes traditionnel qui peut tout aussi bien servir à jouer à la Bataille qu'à la Belote, une partie de la production qu'analyse l'auteur et qu'il qualifie de «jeux d'éditeurs » (ou jeux propriétaires), a pour particularité d'associer étroitement règles et contenus au point d'en devenir inséparables : la règle n'a de sens que rapporté à la matérialité du jeu et inversement. Cette solidarité implique une transformation des usages qui ne supposent dès lors plus, contrairement aux jeux traditionnels, une communauté préexistante pour rendre possibles sa transmission et sa pratique. En même temps que se développe cette association entre contenus et règles, l'auteur remarque le recours fréquent à de mécaniques ludiques associant, comme au Monopoly, hasard et transactions monétaires, mais aussi l'importance des titres empruntant au monde et aux métiers réels (commerce, journalisme, etc.). Cette thématisation du jeu de société marque une seconde rupture : à la différence des jeux traditionnels «où seule la pratique confère un sens à des règles abstraites ", ces nouvelles productions, par le caractère situé de leurs contenus et de leurs mécaniques, deviennent "signifiantes avant même d'avoir pris un sens par le fait d'être joués". Cette imitation du monde social ne signifie pas pour autant une réplication du réel, comme s'y emploie à le démontrer la dernière partie du texte à partir d'une réflexion croisée entre théorie des jeux, jeux de simulation et jeux pédagogiques.

21 Le dernier article est enfin une traduction du texte de Bruce Whitehill (1999), "American Games : a Historical Perspective ", publié dans la revue Board Game Studies. Auteur de plusieurs ouvrages et articles de référence sur le jeu de société (Whitehill, 1992), mais aussi créateur et collectionneur, il propose un important éclairage historique sur l'émergence de l'industrie du jeu de société aux États-Unis. Démarrant au milieu du $19^{e}$ siècle sous une forme artisanale et locale, elle devient au début du siècle suivant une industrie culturelle majeure dont plusieurs acteurs s'imposent sur la scène mondiale: Parker Brothers, Milton Bradley ou plus récemment Hasbro qui a successivement acquis les deux premiers. L'histoire de cette industrie, comme le souligne l'auteur, est complexe. Elle est à la fois traversée par celle des techniques (machines d'impression, apparition de la télévision et de l'électronique), et par celle de la colonisation du continent et des grands évènements mondiaux que reflètent les contenus et les illustrations sur les boites de jeu. Marqué tour à tour par des périodes de guerre, de récession et de prospérité, le jeu de société est un témoin privilégié de l'évolution des loisirs, de la famille et de l'enfance aux États-Unis. Mais cette histoire américaine du jeu de société ne se limite pas au nouveau continent. À travers l'analyse de titres tels que le Monopoly, le Scrabble (Mattel/Hasbro, 1948) ou encore le Trivial Pursuit (Hasbro, 1979), comme s'y emploie l'article, c'est toute une partie de la culture ludique mondiale que ces industries ont contribué à transformer et à façonner. 


\section{BIBLIOGRAPHIE}

AMADIEU T. (2013), Sociologie des jeux de hasard. Croyance et rationalité, Thèse de doctorat, Université Paris-Sorbonne

ARIES P. (1982), « Du sérieux au frivole », in J.C. Margolin, P. Ariès (dir.), Les Jeux à la Renaissance, Paris, Vrin, p. 7-15

BARBIER J.-E. (2012), « Jeu d'édition et identités fictives », ¿ Interrogations ?, n 15, http:// www.revue-interrogations.org/Jeu-d-edition-et-identites

BAYECK R. Y. (2020), « Examining Board Gameplay and Learning : A Multidisciplinary Review of Recent Research », Simulation \& Gaming, vol. 51(4), p. 411-431, https://journals.sagepub.com/doi/ $10.1177 / 1046878119901286$

BECQ DE FOUQUIERES L., (1869), Les jeux des Anciens, Paris, C. Reinwald

BELMAS E. (2006), Jouer autrefois : essai sur le jeu dans la France moderne (XVIe-XVIIIe siècle), Seyssel, Champ Vallon.

BENGHOZI P.-J. et CHANTEPIE P. (2017), Jeux vidéo : L'industrie culturelle du XXIe siècle ?, Paris, Presses de la Fondation nationale des sciences politiques

BERRY V. (2017), « Le renouveau du jeu de société », RLPE, nº 300, p. 124-129

BERRY V. et ROUCOUS N. (2020), «Conception et édition de jeux de société : étude du secteur (de niche) et trajectoires professionnelles », Sciences du jeu, $n^{\circ} 13:$ https://journals.openedition.org/ $\operatorname{sdj} / 2732$

BERRY V. (2021, à paraitre), «Quand le jeu vidéo était un jeu parmi d'autres : le cas du magazine Jeux et Stratégie (1980-90)», in S. Ammouche, A. Blanchet, B.O. Dozo B.O. et M. Triclot (dir.), La presse vidéoludique francophone: panorama et enjeux, Liège, Presses Universitaires de Liège

BICKEL J.-F., LALIVE D’ÉPINAY C. et VOLLENWYDER, N. (2005), « Changement et continuité dans les loisirs : Une comparaison de cohortes ", L'Année sociologique, vol. 55, p. 129-169.

BORZAKIAN M. (2007), « Une géographie de la pratique des échecs en France », Annales de géographie, $\mathrm{n}^{\circ} 655$, p. 291-300

BORZAKIAN M. (2009), « Pour une approche géographique des jeux de plateau », Cybergeo : European Journal of Geography document 462, http://cybergeo.revues.org/index22466.html BORZAKIAN M. (2010), Géographie ludique de la France. Approche spatiale des pratiquants et des fédérations des jeux institutionnels, Thèse de doctorat, Université Paris IV-Sorbonne.

BORZAKIAN M. (2015), « Une géographie de la France ludique. La dimension spatiale des pratiques culturelles ", Annales de géographie, $n^{\circ}$ 701, p. 51-75

BOURGUILLEAU A. (2020), Jouer la guerre : Histoire du wargame, Paris, Passés composés BRODY A. (2011), « L'investissement de l'espace domestique par le jeu d'argent : l'exemple du poker », Revue des Sciences Sociales, $\mathrm{n}^{\circ} 45$, pp. 50-56

BROUSSE C. (2015), « La vie quotidienne en France depuis 1974. Les enseignements de l'enquête Emploi du temps », Economie et Statistique, vol. 478, n 1, p. 79-117

CAÏRA O. (2007), Jeux de rôle : Les forges de la fiction, Paris, CNRS 
CAÏRA O. (2018), « Les dimensions multiples de l'engagement ludique », Sciences du jeu, $\mathrm{n}^{\circ} 10$, https://journals.openedition.org/sdj/1149

CHENU A. et HERPIN N. (2002), « Une pause dans la marche vers la civilisation des loisirs ?», Economie et statistique, $\mathrm{n}^{\circ} 352-353$, p. 15-37

CHOQUET O. et VALDELIÈVRE H. (1988), « Les loisirs 20 ans après : davantage de sorties et de télévision ", Les collections de l'INSEE, nº 148, p. 1-4

COAVOUX S. et GERBER D. (2016), « Les pratiques ludiques des adultes entre affinités électives et sociabilités familiales », Sociologie, vol. 7, p. 133-152

DAUPHRAGNE A. (2008), « Dynamiques ludiques et logiques de genre : Les univers de fantasy », in Brougère G. (dir.), La ronde des jeux et des jouets, Paris, Autrement, p. 43-57

DAVID C. (2016), « Le jeu de rôle sur table : Une forme littéraire intercréative de la fiction ?», Sciences du jeu, $\mathrm{n}^{\circ}$ 6, https://journals.openedition.org/sdj/682

DONNAT O. (2011), «Pratiques culturelles, 1973-2008 : Questions de mesure et d'interprétation des résultats ", Culture méthodes, vol. 2, $\mathrm{n}^{\circ}$ 2, p. 1-12

DUMAZEDIER J. (2018 [1962]), Vers une civilisation du loisir ? [1962], Paris, MKF

DUMONTIER F. et VALDELIÈVRE H. (1989), « Les pratiques de loisir vingt ans après 1967/1987-1988 », Insee Résultats, n 3, p. 3-69

FEYRS D. (2002), « Formes et pratiques du clandestin, joueurs de poker et tripots dans une grande ville de province ", Sociologie du travail, vol. 44, n 2, p. 233-253

FINE G. A. (2002). Shared fantasy : Role-playing games as social worlds, Chicago, Univ. of Chicago Press

FINE G. A. (2015), Players and Pawns. How Chess Builds Community and Culture, Chicago, University of Chicago Press

HENRIOT J. (1989), Sous couleur de jouer : La métaphore ludique. Paris, J. Corti

LEGER J. (1994), « Les dépenses pour les loisirs depuis 1960 », Insee Premiere, vol. n 306, p. 1-4

LIZE W. (2004), « Imaginaire masculin et identité sexuelle. Le jeu de rôles et ses pratiquants », Sociétés contemporaines, $\mathrm{n}^{\circ} 55, \mathrm{p} .43-47$

LEGENDRE F. (2015), Jeu et bibliothèque : pour une conjugaison fertile, Rapport d'enquête, Inspection générale des bibliothèques, Ministère de la culture, https://www.enssib.fr/bibliothequenumerique/documents/65198-jeu-et-bibliotheque-pour-une-conjugaison-fertile.pdf

LHOTE J.M. (1994), Histoire des jeux de société : géométries du désir, Paris, Flammarion

PASTINELLI M., CÔTÉ-BOUCHARD S. et PAPINEAU É. (2011), « Quand le poker cesse d'être un jeu : Pratiques et discours d'aspirants joueurs professionnels ", Ethnologies, n 32(1), 87-111, https:// www.erudit.org/fr/revues/ethno/2010-v32-n1-ethno3988/045214ar/

MURRAY H. (1952), A History of Board Games Other Than Chess, New York, Oxford University Press

OCTOBRE S., DÉTREZ C., MERCKLÉ P. et BERTHOMIER N. (2010), L'enfance des loisirs : Trajectoires communes et parcours individuels de la fin de l'enfance à la grande adolescence. Paris, La Documentation Française

PIEDALLU J. (2012), « Ethnographie de parties clandestines de poker : de l'intérêt pour les sociabilités aux sociabilités par intérêt », communication au forum Jeu et Sociétés organisé par la Française des Jeux, 31 mai 2012, Boulogne-Billancourt 
PÉRIER I. (2017), « Le jeu de rôle, une autre forme de narration sérielle ? », Itinéraires, n² 2016-2, https://journals.openedition.org/itineraires/3453

PETERSON J. (2012), Playing at the world: a history of simulating wars, people and fantastic adventures, from chess to role-playing games, San Diego, Unreason Press

REDON M. (2012), « Gaguère (combat de coqs) et borlette (loterie) : quels enseignements sur Haïti ? ", Géographie et culture, $n^{\circ} 82, n^{\circ} 82$, p. 97-120

REDON M. (2015), « Les combats de coqs à Timor-Leste : un jeu d'argent institutionnalisable? », Sciences du jeu, $\mathrm{n}^{\circ}$ 3, https://journals.openedition.org/sdj/443

REDON M. et LEBEAU B. (2020), Géopolitique des jeux d'argent : Les enjeux d'une mondialisation silencieuse, Paris, Le Cavalier Bleu

ROGERSON M. J., GIBBS M. et SMITH W. (2016), « "I Love All the Bits" : The Materiality of Boardgames ", Proceedings of the 2016 CHI Conference on Human Factors in Computing Systems, p. 3956-3969. https://dl.acm.org/doi/10.1145/2858036.2858433

ROGERSON M. J. et GIBBS M. (2018), « Finding Time for Tabletop : Board Game Play and Parenting », Games and Culture, vol. 13(3), p. 280-300

ROUCOUS N. et BROUGERE G. (1998), « Loisir et éducation. L'apport d'une nouvelle institution : La ludothèque ", Revue française de pédagogie, vol. 124, p. 91-98

TER MINASSIAN H., BERRY V., BOUTET M., COLÓN DE CARVAJAL I., COAVOUX S., GERBER D., RUFAT S., TRICLOT M., ZABBAN V. (2021), La fin du game ? Les jeux vidéo au quotidien, Tours, Presses universitaires François-Rabelais.

THÉRY H. et FLEURY M.-F. (2002), « Le succès mondial du Monopoly », Mappe Monde, $\mathrm{n}^{\circ}$ 66, p. 33-37

TRÉMEL L. (2001), Jeux de rôles, jeux vidéo, multimédia. Les faiseurs de mondes, Paris, P.U.F.

TRESPEUCH M. (2013), « Du monopole à la concurrence sur Internet. L'exemple du marché français des paris en ligne ", Revue française de socio-économie, $n^{\circ} 8$, p. 39-58.

TYNI H. (2020), « Double Duty : Crowdfunding and the Evolving Game Production Network », Games and Culture, vol. 15, $\mathrm{n}^{\circ}$ 2, p. 114-137

WEBER F. (2008), « Une enquête dans l'histoire. Le travail à-côté, apogée d'une culture ouvrière européenne ", in A.-M. Arborio (dir.), Observer le travail. Histoire, ethnographie, approches combinées, Paris, La Découverte, 2008, p. 201-214

WENDLING, T. (2002), Ethnologie des joueurs d'échecs, Paris, P.U.F.

WHITEHILL B. (1992), Games: American Boxed Games and Their Makers, 1822-1992, Radnor, KP Books

WHITEHILL B. (1999), « American Games : A Historical Perspective », Board Game Studies, 2, p. $116-141$

WOODS S. J. (2009), “(Play) Ground Rules: The Social Contract and the Magic Circle”, Observatorio (OBS*) Journal, vol. , 8, p. 204-222

YONNET P. (1999), Travail, loisir. Temps libre et lien social. Paris, Gallimard

ZABBAN V. (2012), « Retour sur les game studies. Comprendre et dépasser les approches formelles et culturelles du jeu vidéo ", Réseaux, $n^{\circ}$ 173-174, p. 137-176

ZABBAN V., TER MINASSIAN H. et NOÛS C. (2020), « Les mondes de production du jeu vidéo : Logiques amateurs, artisanales et industrielles ", Réseaux, n²24, p. 9-29. 


\section{NOTES}

1. Le texte doit beaucoup aux échanges avec Samuel Coavoux et à ses relectures. Qu'il en soit ici remercié.

2. La catégorie regroupe les activités suivantes : jeux de cartes, loto, PMU, pratiques artistiques et certains loisirs numériques (jeux vidéo) et en ligne (jeux sur internet)

3. Dans les deux vagues d'enquête, la même question est posée : «Jouez-vous aux cartes ou à d'autres jeux de société ? ». L'édition de 1987 se révèle cependant plus détaillée concernant les genres de jeux de cartes (belote, bridge...) et de jeux de société (échecs, dames, Monopoly..., jeu de rôles).

4. La difficulté est redoublée par le fait que les méthodes d'échantillonnage changent entre les éditions. D'autres problèmes méthodologiques se posent dans la comparaison, notamment les mutations structurelles de la population en termes d'âge ou de catégories socioprofessionnelles qui peuvent affecter sa composition au cours du temps (Donnat, 2001).

5. Parmi une liste de matériel de loisirs (canne à pêche, guitare, etc.), les enquêtés doivent se positionner et, pour chacun des équipements, indiquer s'ils le possèdent à domicile et s'ils l'utilisent «souvent », « de temps », « rarement» ou « jamais». Une entrée concerne « un jeu d'échecs", une seconde les "autres jeux de société ». Les deux variables sont ici agrégées. Pratiques culturelles des français - 1973-1974, SER - Ministère de la Culture (producteur), ADISP (diffuseur).

6. Rapportées à l'ensemble échantillon, les pratiques fréquentes de jeux de société concernent $18 \%$ de la population.

7. Les mêmes questions que dans l'édition précédente sont posées : "échecs » et " autres jeux de société ». Rapportées à l'ensemble échantillon, les pratiques fréquentes de jeux de société concernent $20 \%$ de la population. Pratiques culturelles des français - 1981, SER - Ministère de la Culture (producteur), ADISP (diffuseur).

8. Dans cette édition, chaque enquêté doit, parmi une série de loisirs, indiquer sa pratique ou non dans l'année et sa fréquence ("souvent », « de temps en temps », " rarement »). Sont interrogées les pratiques suivantes : «jouer aux cartes », «jouer au PMU » «jouer au loto, au Tac-O-Tac, au Tapis Vert ", "Faire des mots croisés » et pour la première fois dans une enquête publique «jouer à des jeux électroniques ». Pratiques culturelles des français - 1988-1989, DEP - Ministère de la Culture (producteur), ADISP (diffuseur).

9. Parmi ceux qui déclarent y avoir joué, $14 \%$ d'entre l'ont fait de façon fréquente, $20 \%$ de temps en temps et $8 \%$ rarement.

10. La liste d'activités de loisirs est sensiblement la même que dans l'édition précédente. La question concernant les jeux de cartes devient "Jouer aux cartes ou à des jeux de société (Scrabble...) ». Les « jeux électroniques » (voir note 14), les jeux de paris sportifs, les mots croisés, le jeux de tirage et de grattage continuent d'être interrogés sous des formulations proches. Les modalités de codage des fréquence changent et deviennent: "régulièrement tout au long de l'année ", " de temps en temps tout au long de l'année ", "seulement pendant les vacances ». Pratiques culturelles des français - 1997-1998, DEP - Ministère de la Culture (producteur), ADISP (diffuseur).

11. Dans l'enquête de 2008, agrégeant les cartes et les jeux de société, la même question est posée et la pratique ne concerne plus que $49 \%$ des enquêtés. La fréquence n'est plus mesurée et certaines activités ludiques comme les paris sportifs et les jeux de grattage disparaissent au profit d'un questionnement détaillé sur les jeux vidéo: usage, fréquences, genres de jeux pratiqués, équipements etc. Pratiques culturelles des français - 2008, DEPS - Ministère de la Culture (producteur), ADISP (diffuseur).

12. Pour la première fois, l'enquête sur les pratiques culturelles des Français interroge de façon détaillée le jeu de société en termes de pratiques et fréquence, mais aussi de genres : "Jeux de 
cartes et de dés (tarot, belote, poker, yam's...) », «Jeux de logique, de déduction et de stratégie (échecs, dames, Rubik's cube, Les aventuriers du rail)», "Jeux de rapidité (Times'up, Jungle speed...) », « Jeux de coopération et de rôles (Loups Garous, Conan, Edge of the empire, etc.) »... 10 catégories de jeux sont ainsi retenues.

13. Le chiffre concerne ici celles et ceux qui ont pratiqué dans l'année au moins un des 10 genres de jeu de société retenus par l'enquête. Le questionnaire interroge également les enquêtés sur le fait d'avoir plus généralement joué « aux cartes, à des jeux de société, à des jeux de chiffres ou de lettres » dans l'année. A cette question plus large, $51 \%$ répondent positivement.

14. Entre 1970 et 2010 certaines activités à dominante traditionnellement masculine comme le bricolage et le jardinage se sont légèrement féminisées en même temps quelques activités mixtes (courses, travaux divers) se sont masculinisées. Le classement des travaux domestiques des «plus traditionnellement féminins au plus masculins" reste cependant stable sur la période, à l'exception de la vaisselle, et demeure aujourd'hui encore très inégalement distribué selon les genres (Brousse, 2015).

15. Dans ses premières occurrences, le terme désigne à la fois les jeux à cristaux liquides de type Game and Watch (Nintendo, 1980), les premiers jeux vidéo sur console, mais certains jeux de société tels que le Simon (M.B., 1978), les échiquiers et les jeux de batailles navales électroniques.

16. Il existe une importante et ancienne tradition de recherche sur les jeux traditionnels en France et dans le monde (De Fouquières, 1868 ; Murray, 1952; Lhote 1994, Belmas, 2006) qui étudie leur origine, leur diffusion, leur évolution dans le temps. Elle demeure très dynamique comme en témoigne l'association et la revue scientifiques Board Games Studies créées au milieu des années 1990.

17. Dans le champ de la géographie, il faut également évoquer les travaux de Marie Redon sur la diffusion et la pratique des jeux d'argents en Haïti et au Timor oriental (Redon, 2012, 2015) et le travail mené avec Boris Lebeau sur la « géopolitique des jeux d'argent » (Redon et Lebeau, 2020). 18. On peut évoquer à titre d'exemple le journal académique dédié au jeu de société créé en 2013, Analog Game Studies.

\section{RÉSUMÉS}

Introduisant le numéro 14 de la revue Sciences $d u$ jeu, cet article propose un état des connaissances sur le jeu de société dans le champ des sciences humaines et sociales. Dans un premier temps, le texte rassemble différentes données statistiques pour comprendre son évolution sur les soixante dernières années. À travers l'étude de plusieurs enquêtes publiques, il met en évidence, au tournant des années 1980, une augmentation des pratiques, une diversification de l'offre et une transformation des publics qui se traduit notamment par une féminisation des usages. Dans un deuxième temps, l'article examine différentes recherches sur le jeu de société pour en souligner deux logiques distinctes: un premier ensemble de travaux s'intéresse au jeu de société en tant qu'indicateur de transformations sociales et démographiques en cours. Plus récent, un second mouvement de recherche fait du jeu de société l'objet central de l'analyse pour en comprendre ses logiques spécifiques en termes d'engagement, d'expérience et d'apprentissage. L'article se termine par une présentation des contributions de ce numéro.

This article introduces issue $n^{\circ} 14$ of the journal Sciences $d u$ jeu by outlining the state of knowledge on board games in the fields of humanities and social sciences. The text first analyzes 
statistical data from public surveys spanning 60 years. Starting in the early 1980s, it highlights how one can observe an increase in practices, a diversification of the games offered and a transformation of the public, resulting particularly in a feminization of uses. We next examine research on board games, highlighting two distinct perspectives. A first set of studies looks at board games as an indicator of ongoing social and demographic transformations. More recently, a second research movement makes board games the central object of analysis in order to understand their specific mechanisms in terms of engagement, experience and learning. The article ends with a presentation of the papers of this issue.

\section{INDEX}

Mots-clés : jeu de société, sociologie des pratiques, évolution des publics

Keywords : board game, sociology of practices, evolution of audiences

\section{AUTEUR}

\section{VINCENT BERRY}

Experice, Université Sorbonne Paris Nord 